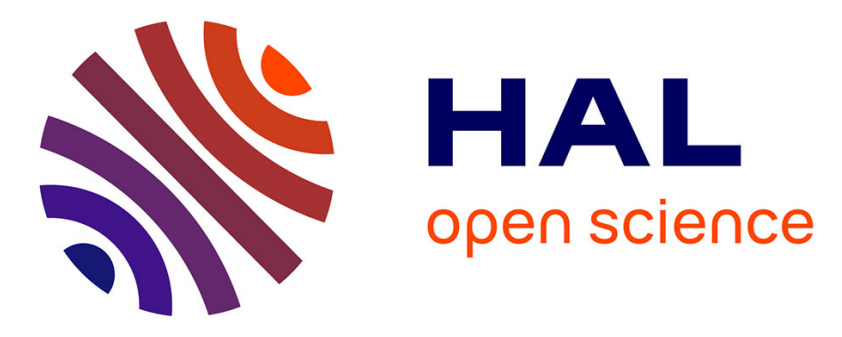

\title{
Plasma induced acceleration and selectivity in strain-promoted azide-alkyne cycloadditions
}

David Warther, Enes Dursun, Marion Recher, Sylvain Ursuegui, Michel

Mosser, Joanna Sobska, Wojciech Krezel, Guilhem Chaubet, Alain Wagner

\section{- To cite this version:}

David Warther, Enes Dursun, Marion Recher, Sylvain Ursuegui, Michel Mosser, et al.. Plasma induced acceleration and selectivity in strain-promoted azide-alkyne cycloadditions. Organic \& Biomolecular Chemistry, 2021, 19 (23), pp.5063-5067. 10.1039/D1OB00529D . hal-03369248

\section{HAL Id: hal-03369248 \\ https://hal.science/hal-03369248}

Submitted on 8 Oct 2021

HAL is a multi-disciplinary open access archive for the deposit and dissemination of scientific research documents, whether they are published or not. The documents may come from teaching and research institutions in France or abroad, or from public or private research centers.
L'archive ouverte pluridisciplinaire HAL, est destinée au dépôt et à la diffusion de documents scientifiques de niveau recherche, publiés ou non, émanant des établissements d'enseignement et de recherche français ou étrangers, des laboratoires publics ou privés. 


\title{
Plasma Induced Acceleration and Selectivity in Strain-Promoted Azide- Alkyne Cycloadditions
}

\author{
David Warther, ${ }^{a}$ Enes Dursun, ${ }^{a}$ Marion Recher, ${ }^{a}$ Sylvain Ursuegui, ${ }^{a}$ Michel Mosser, ${ }^{a}$ Joanna Sobska, ${ }^{b}$ Wojciech \\ Krezel, ${ }^{b}$ Guilhem Chaubet ${ }^{\star a}$ and Alain Wagner ${ }^{\star a}$
}

\begin{abstract}
Strain-promoted azide-alkyne cycloaddition (SPAAC) is an important member of the bioorthogonal reaction family. Over the past decade, much work has been dedicated to the generation of new strained alkynes with improved reactivity. While kinetics studies of SPAAC are often conducted in organic solvents, buffered solutions or mixtures, these media do not reflect the complexity of in vivo systems. In this work, we show that performing SPAAC in human plasma lead to intriguing kinetics and chemoselectivity effects. In particular, we observed that reactions in plasma could be accelerated up to a 70 -fold compared to methanol, and that selective couplings between pair of reagents could be possible in competition experiments. These findings highlight the value of evaluating bioorthogonal reactions in such complex medium, especially when in vivo applications are planned, as unsuspected behaviour can be observed, disrupting the usual rules governing reactivity in simple solvent systems.
\end{abstract}

\section{Introduction}

Bioorthogonal reactions can be described as chemical reactions occurring in a biological environment without interfering with any of its processes or materials. Initially coined by Carolyn R. Bertozzi in the early 2000s, bioorthogonal chemistry has since benefited from many improvements in terms of reactions selectivity and kinetics thanks to the development of more soluble and stable reagents with reduced toxicities. ${ }^{1-9}$ Strain-promoted azidealkyne cycloaddition (SPAAC) is a striking example of such an achievement: reported for the first time in the context of bioorthogonal chemistry in 2004 , it is now considered as an indispensable reaction of the chemical biology toolbox, due to the stability of the resulting triazole products, along with its simplicity of operation, low toxicity and fast kinetic rates. ${ }^{6}$ The latter characteristic is a crucial parameter to take into account for in vivo applications, as the reaction has to be rapid in order to occur at low concentrations, before reactants are metabolized or excreted. Major efforts have thus been dedicated to optimizing SPAAC kinetic rates via the development of more reactive alkyne through the modulation of stereoelectronic parameters. ${ }^{10-14}$ In this race for the ideal bioorthogonal reagent, the effect of the surrounding biological environment in which the reaction takes place has been somehow overlooked. Even though it is well known that solvent effects strongly influence cycloaddition rates, ${ }^{15}$ most studies have been conducted in organic-aqueous solvent mixtures, those systems being often considered as representative of the more complex biological media. Interestingly however, the group of Carolyn R. Bertozzi reported the unexpected reactivity of several alkynes probes when using SPAAC to label azidefunctionalized glycans in living mice. ${ }^{16}$ Despite comparable labelling efficiencies in vitro, in coherence with similar second-order rate constants of the parent cyclooctynes in model reactions, ${ }^{17-19}$ several alkyne probes gave markedly different results when tested in vivo, suggesting that the chemical structure of the probe itself was a key parameter of its in vivo reactivity. In the same vein, Robillard and coworkers described the use of SPAAC reactions for the modification of azide-containing antibodies in live mice and showed that serum proteins - and especially albumin - had a negative impact on the reaction kinetics, due to their strong interactions with alkynes, making the latter less available to react with the antibodies' azide groups. ${ }^{20}$ Altogether, these results support the hypothesis that the outcome of bioorthogonal reactions in vivo cannot be predicted solely based on the parent alkyne's reactivity in model reactions conducted in simple solvent systems. As more complex parameters may play a substantial role, new in vitro models are thus needed to mimic this environment in order to decipher the underlying rules governing the fate of bioorthogonal reactions in complex media.

\section{Results and discussion}

Building on a previous work in which we reported the in vivo neutralization of the azido derivative $\mathbf{1}$ of the anticoagulant warfarine by a SPAAC reaction with

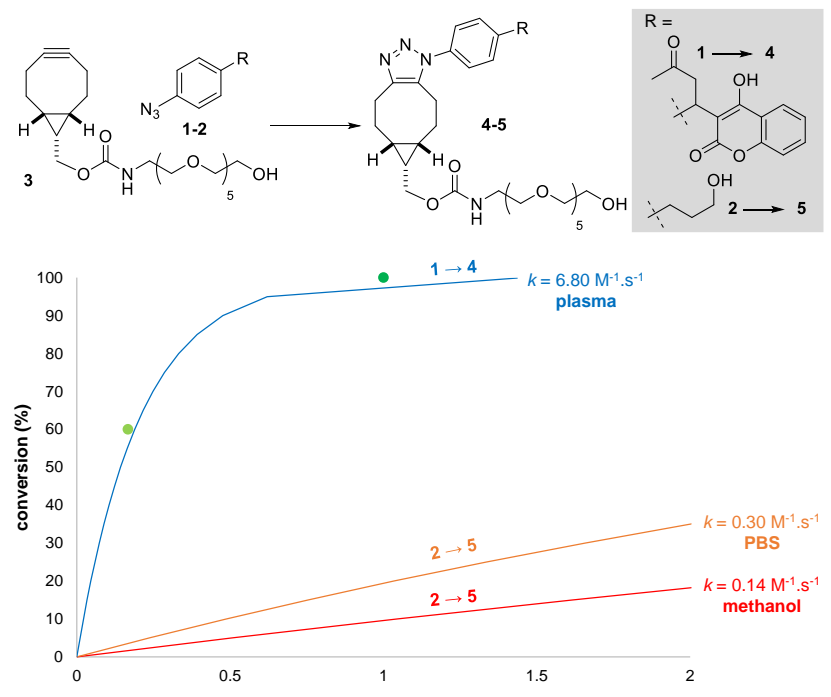

Figure 1. Differences in kinetics between strain-promoted azide-alkyne cycloadditions run either in classical solvent systems or in plasma. The green dots represent the conversion observed in-vivo. 
bicyclononyne (BCN) 3, ${ }^{15}$ we started by evaluating human plasma as a surrogate for in vivo models. It became quickly apparent that rate constants were extremely similar between in vivo and plasma media, and in sharp contrasts with those measured with the model azide 2 in both methanol and phosphate buffered saline (PBS) solutions (Figure 1 and S2). While such strong discrepancies underlined a major influence of the medium on SPAAC kinetics, the fact that we were comparing two different systems ( $\mathbf{1} \rightarrow \mathbf{4}$ in plasma and in mice vs $\mathbf{2} \rightarrow \mathbf{5}$ in PBS and methanol) prevented us from drawing this definitive conclusion.

In order to probe the influence of plasma on the outcome of SPAAC reactions, we decided to perform a systematic study by measuring the cross reactivity of pairs of alkyne / azide under standardized conditions and determining their corresponding second order kinetic rate constants. We designed a coherent series of $\mathrm{BCN}$ derivatives, all synthesized from the simple BCN alcohol 6: one bearing an apolar dioxole group (7) and three equipped with polar moieties, displaying either a permanent charge at physiological $\mathrm{pH}$ (8 and 9) or possessing a short and neutral oligoethyleneglycol (OEG) chain (3). Each of these $\mathrm{BCN}$ probes was paired with a counterpart azide equipped with similar functional groups $(\mathbf{2}, \mathbf{1 0 - 1 2})$. These moieties were chosen with the aim of modulating the biophysical properties of the reagents without affecting their intrinsic chemical reactivity. Each pair was then reacted in equimolar conditions at a concentration of $0.1 \mathrm{mM}$ in human plasma. This standard concentration was chosen since it was consistent with the maximum plasmatic concentration of PEG 3 measured after sub-cutaneous injection (52 mg. $\mathrm{kg}^{-1}$ ) in mice. ${ }^{15}$ In methanol, the concentration had to be increased to $5 \mathrm{mM}$ in order to start seeing product formation within the two hours timeframe of a classical experiment. In each case, 1,7dihydroxynaphthalene was used as an internal standard in order to improve the precision of our analytical method, and the reactions were monitored by HPLC at various time points, following both azide disappearance and triazole product formation (see SI). As all BCN and azide compounds evaluated in these experiments were perfectly stable in plasma and since the expected triazole was the only product formed in all reactions, the second-order kinetic constant of the different SPAAC reactions was calculated by plotting the evolution of the inverse of concentration of the azide component as a function of time according to the following equation:

$$
1 /[A]=1 /[A]_{0}+k t
$$

where $[A]_{0}$ is the initial molar concentration of the azide reactant; $[A]$, the molar concentration of the azide reactant at a given time; $t$, the time in seconds; and $k$, the secondorder kinetic constant expressed in $\mathrm{M}^{-1} \cdot \mathrm{S}^{-1}$ (see ESI for details about calculations).

We found that SPAAC kinetics were quite slow in methanol $\left(\leq 0.57 \mathrm{M}^{-1} \mathrm{~s}^{-1}\right)$ and with little variations between each $\mathrm{BCN}$ / azide pair evaluated $\left(\sigma \sim 0.17 \mathrm{M}^{-1} \mathrm{~s}^{-1}\right)$, clearly suggesting that the reactants structure had little to no influence on the outcome of SPAAC reactions in the organic solvent (Figure 2). In plasma however, reactions were found to be greatly accelerated compared to methanol, up to a 70-fold with second-order kinetic constant values culminating at $\sim 19 \mathrm{M}^{-}$ $1 \mathrm{~s}^{-1}$. In addition, striking differences in reactivity were found between the different reactant pairs depending on their structure and skeleton $\left(\sigma \sim 4.73 \mathrm{M}^{-1} \mathrm{~s}^{-1}\right)$. One notable exception concerned carboxylate-bearing azide 12, whose reactivity was barely impacted by either the $\mathrm{BCN}$ reagent or the medium composition. At this stage, it is important to reiterate that reactions were fifty times more concentrated
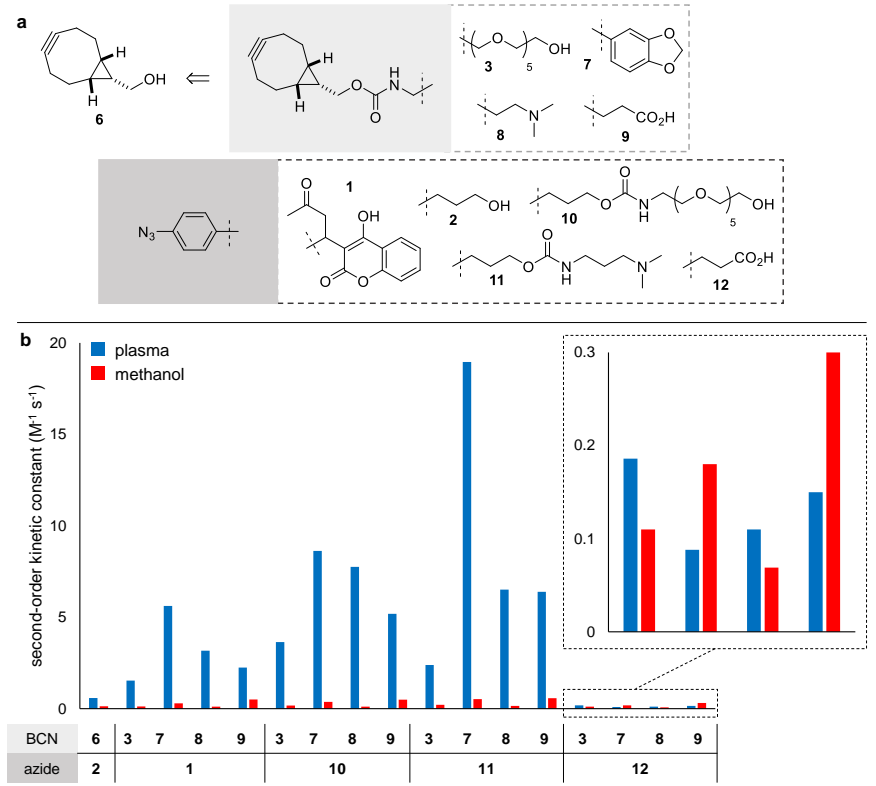

Figure 2. Influence of azides and cyclooctynes structures on the rate of SPAAC reactions; (a) structures of the different BCN and azide derivatives utilized in the study; (b) representation of the different second-order kinetic constants measured in plasma (100 $\mu \mathrm{M})$ and in methanol ( $5 \mathrm{mM}$ ) for the SPAAC reactions between different $\mathrm{BCN}$-azide pairs. These values represent the average of measurements made in triplicates $(n=3)$.

in methanol than in plasma ( $5 \mathrm{mM}$ vs $0.1 \mathrm{mM})$, and differences in conversion rates between the two media were logically even more pronounced when reactions were conducted at $0.1 \mathrm{mM}$ in both cases (see ESI).

Lastly, comparison between kinetics in PBS and methanol showed that while reactions between azide $\mathbf{1 1}$ and all BCN probes were accelerated in water - a feature previously reported in the literature -, this was to a much lesser extent than in plasma, highlighting that the aqueous nature of the reaction medium cannot be the sole explanation for the effects observed (see ESI). Taken altogether, these data demonstrate that the reactivity of a strained alkyne or an azide in plasma can be strongly modulated by the chemical structure of the moieties appended to it. Consequently, by performing SPAAC in plasma instead of classical solvent systems, it should be possible in theory to discriminate between two azides or two BCN and attain unprecedented selectivity. 

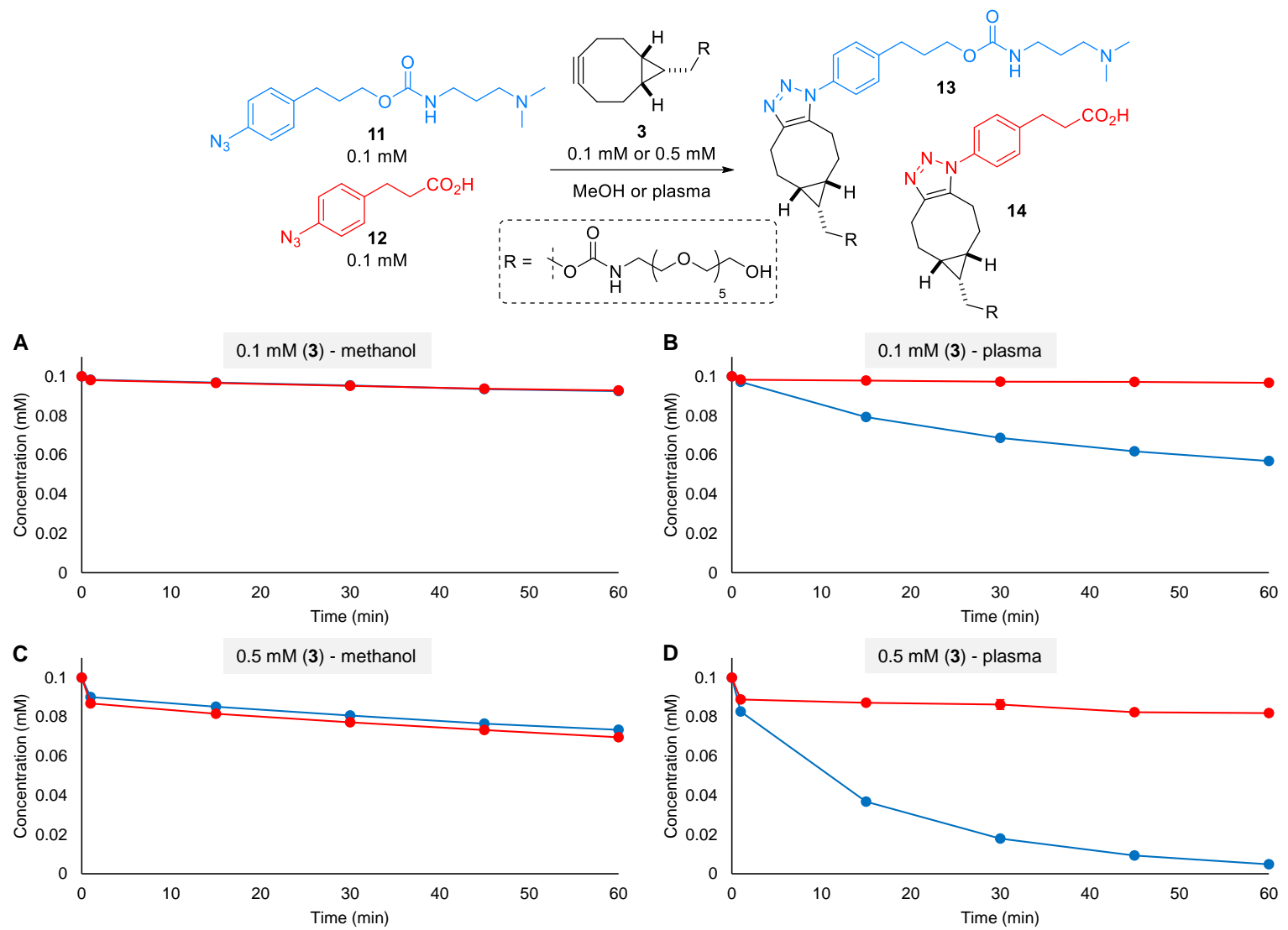

Figure 3. Competition experiments between azides 11 and 12 for reaction with BCN 3 via SPAAC and the influence of medium composition and reactants concentration in the observed conversion. Measurements were done in triplicates $(n=3)$.

We thus selected BCN-azide pairs 3-11 and 3-12, whose corresponding SPAAC showed similar kinetic rates in methanol $\left(0.21 \mathrm{M}^{-1} \cdot \mathrm{s}^{-1}\right.$ and $0.11 \mathrm{M}^{-1} \cdot \mathrm{s}^{-1}$, respectively) but substantial differences in plasma $\left(2.39 \mathrm{M}^{-1} \cdot \mathrm{s}^{-1}\right.$ and $0.19 \mathrm{M}$ $\left.{ }^{1} . s^{-1}\right)$ and performed competition experiments. As expected, both azides were found to be equally reactive at $0.1 \mathrm{mM}$ in methanol, leading to an equimolar mixture of triazoles 13 and 14 (Figure 3, A). As anticipated also at this concentration and in this solvent, less than $10 \%$ conversion was observed after 60 minutes. In plasma however, azide 11 predominantly and quickly reacted, leading to $\sim 40 \%$ conversion to the expected triazole product 13 after 60 minutes, while azide 12 did not participate in any visible reaction (blue line and red line, respectively; Figure 3, B). This illustrates that selectivity between two nearly identical SPAAC can be attained just by switching from classical solvent to plasma.

To evaluate the potential applications of such behaviour in the context of in vivo chemistry, where SPAAC are usually conducted with an excess of strained alkyne, the concentration of $\mathrm{BCN} 3$ was increased to $0.5 \mathrm{mM}$ while keeping the same concentrations in azides 11 and 12 (i.e. $0.1 \mathrm{mM}$ ), now making them the limiting reactants. As expected, faster reactions were observed in both methanol and plasma (Figure 3, C and D). Despite this acceleration, the same trend as before was still observed. No differences between 11 and 12 were noticed in methanol at $0.5 \mathrm{mM}$ and poor conversion was detected for both substrates, while an even better level of selectivity between azides $\mathbf{1 1}$ and 12 was found in plasma: while carboxylate derivative 12 gave less than $20 \%$ conversion to triazole 14 , the ammonium derivative $\mathbf{1 1}$ gave almost complete conversion to 13 after 60 minutes.

To understand better the underlying effects at stake in these reactions, we conducted plasma protein-binding assays to determine the fraction of free azides and BCNs in plasma. Utilizing the equilibrium dialysis method (see ESI for detailed procedures), which had previously allowed us to determine the percentage of warfarin azide 1 bound

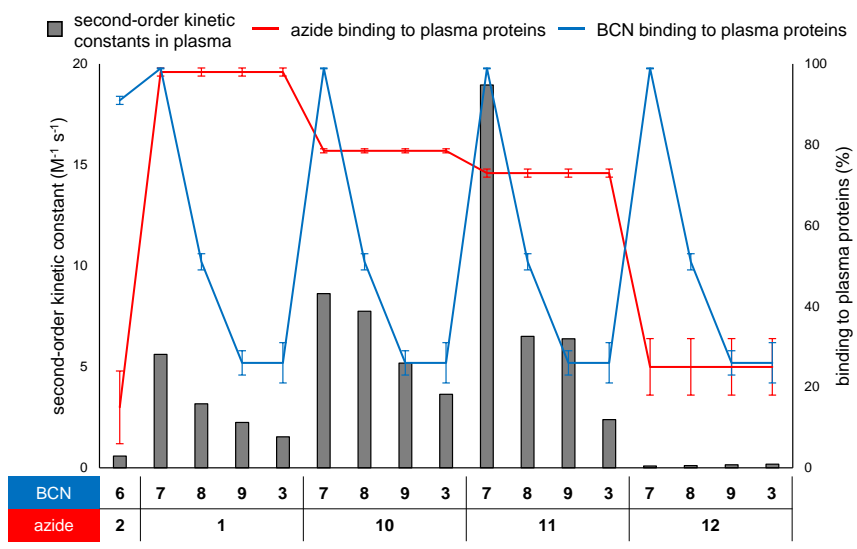

Figure 4. Plasma proteins-binding values for all $\mathrm{BCN}$ and azide compounds evaluated (curves) plotted together with the previously determined second order kinetic constants of SPAAC reactions in plasma (histograms). Measurements were done in triplicates $(n=3)$. 
to plasma proteins to be $94 \%,{ }^{15}$ we found sharp variations between our set of compounds (Figure 4). Binding values of azides were shown to range from $15 \%$ for compound 2 to $98 \%$ for 1 . A comparable variation was also observed for BCN, going from $26 \%$ for both 3 and $\mathbf{9}$, to almost $99 \%$ for compound 7. This plasma-protein binding affinity did not seem to be directly correlated to the functional groups equipping the SPAAC reagents: primary alcoholcontaining azide 2 and BCN 6 gave completely opposite values (15\% and $91 \%$, respectively), similar to what was observed with OEG-functionalized BCN 3 (26\%) and azide $10(78.5 \%)$. When comparing binding values and second order kinetic constants in an attempt to find a correlation between the two sets of values, we observed that the most bound azides (i.e. 1, 10 and 11) systematically gave the highest SPAAC kinetics, while the less bound azides 2 and 12 led to mediocre rate constants. In addition, for a given azide, SPAAC kinetics appeared to be closely related to and modulated by the percentage of proteins-bound BCNs, with compound 7 giving always the best results. These findings relate well to the previously mentioned study from the Robillard group, who also reported that serum proteins were strongly interacting with alkynes. ${ }^{20}$ While in the latter case this proved to be detrimental to SPAAC reactions with azide groups borne by bulky immunoconjugates, a similar effect might be beneficial for reactions between compounds of lower molecular weights. Even though other factors might also be at stake, such as the compounds hydrophobicity or flexibility, the binding of reagents to surrounding proteins seems to have a major influence on the acceleration of SPAAC in plasma. Albeit counterintuitive, local increase of concentration at the surface of a plasma protein could facilitate interactions between azides and BCN in such dilute medium and result in the acceleration of SPAAC.

To validate this hypothesis, we reacted our previously described model system - BCN 3 and azide $1-{ }^{15}$ in PBS in the presence of increasing amounts of human serum albumin (HSA, see ESI for more details), the most abundant protein in human plasma. ${ }^{21}$ Gratifyingly, the second-order kinetic constant determined at plasma-like concentrations of HSA in PBS (i.e. 50 g. $\mathrm{L}^{-1}$ ) was found to be in agreement with that measured in human plasma (1.66 M $\mathrm{M}^{-1} \cdot \mathrm{s}^{-1}$ vs $1.55 \mathrm{M}^{-1} \cdot \mathrm{s}^{-1}$, respectively). While this observation tends to support the hypothesis that protein binding is responsible for the acceleration of SPAAC in human plasma, we also found that the highest rate acceleration was observed in relatively diluted HSA solutions (3.33 $\mathrm{M}^{-1} . \mathrm{s}^{-1}$ at $2.5 \mathrm{mg} \cdot \mathrm{mL}^{-1}$ of HSA in PBS). This shows that plasma proteins and their concentrations exert a strong influence on the kinetics of SPAAC. Understanding better the factors governing this peculiar behaviour and the role of these "participating spectators" in biological media could open up new directions in chemical biology.

\section{Conclusions}

In conclusion, we showed the rate enhancement of various SPAAC reactions conducted in plasma compared to methanol and PBS. Functional groups borne by different $\mathrm{BCN}$ and azide moieties were found to have a profound impact on the reactions' kinetic constants. We took advantage of this effect to successfully conduct competition experiments, where only one of two azide compounds reacted with a given BCN. To the best of our knowledge, this is the first report of an in situ selectivity in plasma between two identical bioorthogonal reactions, only caused by the chemical structure of the reactants. Exploring interactions between biological medium and reactive partners of SPAAC reaction and including this medium as an influential reaction partner should be taken into account when developing or optimizing bioorthogonal reactions. Such phenomena open unexpected perspectives in the development of effective orthogonal pairs based on a single reactive core and expand the in vivo chemistry toolbox while alleviating the need for the production of new families of strained alkynes.

\section{Author Contributions}

DW took care of investigation, methodology, validation, visualisation and writing the original draft of this manuscript.

ED took care of methodology and validation

MR and SU took care of data curation \& early work and investigation.

MM and JS took care of methodology.

WK took care of conceptualisation, funding acquisition, resources, supervision and reviewing \& editing the final manuscript.

GC took care of funding acquisition, project administration, supervision, visualisation, writing the original draft and reviewing \& editing the final manuscript.

AW took care of conceptualisation, funding acquisition, project administration, resources, supervision and reviewing \& editing the final manuscript.

\section{Conflicts of interest}

There are no conflicts to declare.

\section{Acknowledgements}

The authors would like to thank the "Agence Nationale de la Recherche" for financial support (DW, ED).

\section{Notes and references}

1 E. Kim and H. Koo, Chem. Sci., 2019, 10, 7835-7851.

2 E. M. Sletten and C. R. Bertozzi, Angew. Chem. Int. Ed., 2009, 48, 6974-6998.

3 C. P. Ramil and Q. Lin, Chem. Commun., 2013, 49, 11007-11022.

4 J. A. Prescher and C. R. Bertozzi, Nat. Chem. Biol., 2005, 1, 13-21.

5 E. M. Sletten and C. R. Bertozzi, Acc. Chem. Res., 2011, 44, 666-676. 
6 C. G. Gordon and C. R. Bertozzi, in Chemoselective and Bioorthogonal Ligation Reactions, John Wiley \& Sons, Ltd, 2017, pp. 417-457.

7 K. Lang and J. W. Chin, ACS Chem. Biol., 2014, 9, 16-20.

8 B. L. Oliveira, Z. Guo and G. J. L. Bernardes, Chem. Soc. Rev., 2017, 46, 4895-4950.

9 D. M. Patterson, L. A. Nazarova and J. A. Prescher, ACS Chem. Biol., 2014, 9, 592-605.

10 M. F. Debets, S. S. van Berkel, J. Dommerholt, A. (Ton) J. Dirks, F. P. J. T. Rutjes and F. L. van Delft, Acc. Chem. Res., 2011, 44, 805-815.

11 G. de Almeida, E. M. Sletten, H. Nakamura, K. K. Palaniappan and C. R. Bertozzi, Angew. Chem. Int. Ed., 2012, 51, 2443-2447.

12 T. Harris, G. dos P. Gomes, S. Ayad, R. J. Clark, V. V. Lobodin, M. Tuscan, K. Hanson and I. V. Alabugin, Chem, 2017, 3, 629-640.

13 M. King, R. Baati and A. Wagner, Chem. Commun., 2012, 48, 9308-9309.

14 J. Dommerholt, S. Schmidt, R. Temming, L. J. A. Hendriks, F. P. J. T. Rutjes, J. C. M. van Hest, D. J. Lefeber, P. Friedl and F. L. van Delft, Angew. Chem. Int. Ed., 2010, 49, 9422-9425.

15 S. Ursuegui, M. Recher, W. Krężel and A. Wagner, Nat. Commun., 2017, 8, 1-8.

16 P. V. Chang, J. A. Prescher, E. M. Sletten, J. M. Baskin, I. A. Miller, N. J. Agard, A. Lo and C. R. Bertozzi, Proc. Natl. Acad. Sci., 2010, 107, 1821-1826.

17 N. J. Agard, J. M. Baskin, J. A. Prescher, A. Lo and C. R. Bertozzi, ACS Chem. Biol., 2006, 1, 644-648.

18 N. J. Agard, J. A. Prescher and C. R. Bertozzi, J. Am. Chem. Soc., 2004, 126, 15046-15047.

19 E. M. Sletten and C. R. Bertozzi, Org. Lett., 2008, 10, 3097-3099.

20 S. M. van den Bosch, R. Rossin, P. Renart Verkerk, W. ten Hoeve, H. M. Janssen, J. Lub and M. S. Robillard, Nucl. Med. Biol., 2013, 40, 415-423.

21 G. Fanali, A. di Masi, V. Trezza, M. Marino, M. Fasano and P. Ascenzi, Mol. Aspects Med., 2012, 33, 209-290. 